\title{
Outcome of patients $\geq 60$ years of age after alcohol septal ablation for hypertrophic obstructive cardiomyopathy
}

\author{
Denisa Jahnlová ${ }^{1}$, Pavol Tomašov ${ }^{1}$, Radka Adlová ${ }^{1}$, Jaroslav Januška², Jan Krejčín ${ }^{3}$, \\ Maciej Dabrowski ${ }^{4}$, Josef Veselka ${ }^{1}$
}

\author{
${ }^{1}$ Department of Cardiology, Charles University in Prague, $2^{\text {nd }}$ Faculty of Medicine \\ and Motol University Hospital, Prague, Czech Republic \\ 2Department of Cardiology, Heart Centre, Hospital Podlesí a. s., Třinec, Czech Republic \\ ${ }^{3}$ St. Anne's University Hospital, Brno, Czech Republic \\ ${ }^{4}$ National Institute of Cardiology, Warsaw, Poland
}

Submitted: 25 January 2017

Accepted: 29 May 2017

Arch Med Sci 2019; 15 (3): 650-655

DOI: https://doi.org/10.5114/aoms.2019.84735

Copyright (c) 2019 Termedia \& Banach

\begin{abstract}
Introduction: The outcome of patients $\geq 60$ years of age after alcohol septal ablation (ASA) for obstructive hypertrophic cardiomyopathy (HCM) remains unresolved. We sought to determine the long-term survival and the causes of death in this population.

Material and methods: We enrolled 156 consecutive patients (69 \pm 6 years, $69 \%$ women, follow-up: $4.8 \pm 3.5$ years) who underwent ASA at $\geq 60$ years of age.

Results:The 30 -day mortality rate was $1.3 \%$. At the last check-up, $81 \%$ of patients were in New York Heart Association class $\leq 2$ and $76 \%$ had a left ventricular outflow tract gradient (LVOG) $\leq 30 \mathrm{~mm} \mathrm{Hg}$. A total of 39 patients died $(51 \%$ of cardiovascular causes, $44 \%$ of non-cardiovascular causes, $5 \%$ of unknown causes) during the 734 patient-years. The annual sudden mortality, the sudden mortality and the all-cause mortality rates were $0.5 \%$, $1.1 \%$, and $4.8 \%$, respectively. The all-cause mortality was higher compared to the age- and sex-matched general population $(p=0.002)$.

Conclusions: Alcohol septal ablation was safe and effective in the long-term follow-up. We observed a reduced life expectancy compared to the age- and sex-matched general population. Mortality was almost equally due to cardiovascular and non-cardiovascular causes of death.
\end{abstract}

Key words: hypertrophic cardiomyopathy, sudden cardiac death, survival.

\section{Introduction}

Hypertrophic cardiomyopathy (HCM) is a genetic disease defined by the presence of increased left ventricular wall thickness (LVWT) in the absence of abnormal loading conditions sufficient to cause the observed abnormality [1-4]. Patients with HCM are at risk of sudden death (SD), progressive heart failure, and other cardiovascular complications [5]. Recently published data based on an unselected population of HCM patients $\geq 60$ years of age suggested that older patients are at low risk of SD and the mortality rate of these patients is mainly influenced by other causes of death $[6,7]$. Left ventricular outflow tract (LVOT) obstruction is

\author{
Corresponding author: \\ Denisa Jahnlová MD \\ Department of Cardiology \\ University Hospital Motol \\ $\checkmark$ Úvalu 84 \\ 15006 Praha 5 \\ Czech Republic \\ Phone: +420224434901 \\ Fax: +420224434920 \\ E-mail: denisajahn@gmail. \\ com
}


an established independent risk factor for progression of heart failure and of cardiac death in HCM patients [8]. In the treatment of symptomatic patients with obstructive HCM who are refractory to medical therapy, alcohol septal ablation (ASA) or surgical myectomy is recommended $[1,2]$.

Despite early concerns about the potential arrhythmogenicity of the ablation scar, recent studies have shown acceptable periprocedural and long-term results of patients treated with ASA [9-14], comparable to those treated with surgical myectomy [15-18].

Alcohol septal ablation seems to be a preferable therapeutic approach, in patients $\geq 60$ years of age with obstructive HCM, because of higher surgical risk [1, 2, 19]. However, the long-term outcome of this specific group of patients remains unresolved. Therefore, in this study, we sought to determine the long-term survival, major causes of death, and the sudden mortality rate in patients $\geq 60$ years of age with obstructive HCM after ASA.

\section{Material and methods}

\section{Study population}

A total of 332 patients with obstructive HCM (58 \pm 13 years, 55\% women) underwent ASA between April 1998 and March 2014 in four European cardiovascular centers and were enrolled into their databases. Within this group, we identified 156 patients aged $\geq 60$ years of age at the time of ASA (69 \pm 6 years, $69 \%$ women) who met the inclusion criteria of this study. Some of these patients were included in previous reports.

The diagnosis of obstructive HCM was based on the echocardiographic demonstration of a hypertrophied non-dilated left ventricle (maximum LVWT $\geq 15 \mathrm{~mm}$ ) and the presence of a maximal left ventricular outflow tract gradient (LVOG) of $\geq 50 \mathrm{~mm} \mathrm{Hg}$ at rest or during the provocative maneuvers. The baseline LVWT measurement was defined as maximal septal thickness in the basal septum while the post-procedural LVWT was determined by measuring the thinnest part of the intervened basal septum. Alcohol septal ablation was performed only in patients with obstructive HCM that remained highly symptomatic despite maximal tolerated dosage of the optimal medical therapy.

All therapeutic options were discussed with each patient and the decision was made after careful explanation of the risks and benefits of each alternative. Written informed consent was provided by each patient and the local ethics committee approved the study protocol.

\section{Interventional procedure and follow-up}

Details of the ASA technique were published previously $[20,21]$. Patients were observed in the coronary care unit for $\geq 2$ days after the procedure and the temporary pacemaker was removed 2-3 days after ASA if no episode of atrioventricular block occurred. In patients with persistent or recurrent complete heart block, a permanent pacemaker was implanted prior to discharge from the hospital. All patients underwent a clinical, electrocardiographic, and echocardiographic check-up at 3-6 months after ASA and once a year thereafter. In patients with an implanted pacemaker or implantable cardioverter-defibrillator (ICD), the device memory and function were assessed, and the history of appropriate and inappropriate discharges was recorded.

The survival of patients was continuously checked in the National Database of the Departed in each of the participant countries. For the deceased patients who died outside of the study institutions, interviews or mail communication with their next of kin was performed to find out the cause of death.

\section{Definitions}

The primary endpoint was all-cause mortality and the secondary endpoint was a composite of all-cause mortality or appropriate ICD discharge. We compared the incidence of all-cause mortality, as well as the all-cause mortality including the first appropriate ICD discharge, with the expected survival of the age- and sex-matched general population.

The causes of death were classified as non-cardiovascular causes of death and cardiovascular causes of death (including heart failure, stroke, procedure-related death, SD, and appropriate ICD discharge). Sudden death was defined as an unexpected natural death within $1 \mathrm{~h}$ after a witnessed collapse in a previously stable patient. An appropriate ICD discharge was defined as the first device intervention after ASA triggered by ventricular tachycardia (VT) or ventricular fibrillation (VF). Death within 30 days after ASA at least partially attributable to ASA was considered ASA-related. Any death that could not clearly be attributed to one of these groups was classified as a death of unknown cause.

\section{Statistical analysis}

Data are presented as mean \pm standard deviation. The $\chi^{2}$ test, Student's $t$-test, and Kaplan-Meier survival analysis were used as appropriate. Mortality rates were calculated for each individual and combined to form an expected summary curve for the general population. Expected survival was calculated according to age- and gender-specific mortality rates obtained from the Demographic Yearbook of the Czech Republic (http://www.czso.cz/ 
csu/ 2012edicniplan.nsf/engpubl). Expected and observed mortality rates were compared using the one-sample log-rank test, which provides a standardized mortality ratio and the $95 \%$ confidence interval $(\mathrm{Cl})$. Cox proportional hazards regression was used to identify the predictors of all-cause mortality, cardiovascular mortality, and SD events. Clinical variables with a potential effect on patient prognosis were chosen for univariable analysis and included age, baseline and residual dyspnea, baseline and residual LVOG, baseline and residual maximum LVWT, and baseline and residual left ventricular end-diastolic diameter (LVEDD). Variables with a $p<0.15$ were then entered into a multivariable analysis, which was performed using a backward stepwise multiple Cox regression. A $p<0.05$ was considered statistically significant. The statistical software GraphPad Prism v. 6.05 (GraphPad Software, La Jolla, CA) was used.

\section{Results}

\section{Baseline characteristics}

A total of 156 patients underwent ASA between 60 and 86 years of age (69 \pm 6 years); among them, 108 (69\%) patients were women. Baseline echocardiographic and clinical characteristics of the study population are summarized in Table I. Of these patients, 141 (90\%) suffered from dyspnea of New York Heart Association (NYHA) functional class $\geq 3$, while a combination of dyspnea and angina was present in 106 (75\%) patients, and 33 (23\%) patients experienced repeated syncope. Five patients had been implanted with a permanent pacemaker and 5 patients with an ICD prior to ASA. One patient had experienced an appropriate ICD discharge before ASA.

Mean alcohol injection volumes for the ASA procedure were $1.7 \pm 0.8 \mathrm{ml}$ with a subsequent CK-MB peak of $3.1 \pm 2.3 \mu \mathrm{kat} / \mathrm{l}(\mathrm{ULN}=0.4 \mu \mathrm{kat} / \mathrm{l})$. Alcohol septal ablation was combined with percutaneous coronary intervention in $9(5.8 \%)$ patients.

\section{Procedural outcomes}

Four $(2.6 \%)$ patients died during the first 1-month follow-up. Two of them died early after ASA; 1 patient died of cardiac tamponade and 1 patient died of pulmonary embolism. The other 2 patients died of cancer and stroke during the 1-month follow-up after being discharged from the hospital (in-hospital mortality was 1.3\%; 30day ASA-related mortality was 1.3\%).

Post-procedural transient complete heart block was identified in 38 (24.4\%) patients and a permanent pacemaker for a persistent or recurrent complete heart block was implanted in 18 of these patients (11.5\% of total study population). Post-procedural sustained VT or VF requiring urgent cardioversion/defibrillation was observed in 5 (3.2\%) patients.

\section{Long-term outcomes}

None of the 156 patients were lost to follow-up. The mean duration of follow-up was $4.8 \pm 3.5$ years. Clinical and echocardiographic outcomes are summarized in Table I. At the last clinical check-up, a total of 126 (81\%) patients suffered from dyspnea of NYHA class $\leq 2$ and a total of 119 (76\%) patients had a maximal LVOG $\leq 30 \mathrm{~mm} \mathrm{Hg}$ at rest or during the provocative maneuvers. During the follow-up, 2 (1.3\%) patients underwent myectomy and 6 (3.9\%) patients underwent repeated ASA. Eight patients after ASA combined with percutaneous coronary intervention are still alive and 1 patient died of cancer 4 years after the procedure. Eight patients (5.2\%, mean follow-up: $4.9 \pm 2.5$ years) underwent an ICD implantation during the follow-up. The decision was based on current guidelines [1, 2]. Two of them experienced an appropriate ICD discharge during the study period. Additionally, another two patients with an ICD implanted before ASA also experienced an appropriate ICD discharge during the follow-up.

Table I. Clinical and echocardiographic characteristics at baseline and follow-up

\begin{tabular}{|lccc|}
\hline Parameter & Baseline & Follow-up & $P$-value \\
\hline Dyspnea, NYHA class & $3 \pm 0.5$ & $1.8 \pm 0.7$ & $<0.001$ \\
\hline Angina, CCS class & $1.4 \pm 1.2$ & $0.4 \pm 0.7$ & $<0.001$ \\
\hline Episodes of syncope (\%) & 21 & 6 & 0.002 \\
\hline LVOG [mm Hg] & $76 \pm 44$ & $22 \pm 22$ & $<0.001$ \\
\hline LVEDD [mm] & $43 \pm 5.5$ & $47 \pm 5.7$ & $<0.001$ \\
\hline Left ventricular EF (\%) & $71 \pm 10$ & $68 \pm 10$ & 0.025 \\
\hline Baseline maximum LVWT [mm] & $20 \pm 3.6$ & $15 \pm 4.6$ & $<0.001$ \\
\hline
\end{tabular}

Data are presented as mean \pm standard deviation. NYHA - New York Heart Association, CCS - Canadian Cardiovascular Society, LVOG left ventricular outflow tract gradient, LVEDD - left ventricular end-diastolic diameter, EF - ejection fraction, LVWT - left ventricular wall thickness. 
Overall, a total of 4 (2.6\%) patients experienced an appropriate ICD discharge during the follow-up. Mean time between ASA and the ICD discharge was $4 \pm 1.7$ years.

Thirty-six (23\%) patients died during the 747 patient-years ( $81 \%$ women) and the all-cause mortality rate was $4.8 \%$ per year. In these patients, ASA was performed at an average age of $69.7 \pm 5.8$ years, the mean survival was $4.2 \pm 3.8$ years, and the mean age at death was $74 \pm 6.2$ years. Causes of death are summarized in Figure 1. Four (2.6\%) other patients experienced an appropriate ICD discharge and $1(0.6 \%)$ patient experienced an appropriate ICD discharge and died later during the follow-up. Considering the first appropriate ICD discharge as an equivalent of SD, 39 (25\%) patients died during the 734 patient-years with an all-cause mortality rate of $5.2 \%$ per year. The mean survival was $4.1 \pm 3.6$ years and the mean age at death was $74 \pm 6.1$ years. All patients who died were divided into subgroups according to the cause of death. Mortality events from cardiovascular, non-cardiovascular, and unknown causes of death were $51 \%, 44 \%$, and $5 \%$, respectively.

Cardiovascular mortality events occurred in 20 patients $(51 \%$ of all mortality events, $13 \%$ of all patients, mean age at death: $72.5 \pm 4.5$ years), with an annual mortality rate of $2.7 \%$. Among these 20 patients, 4 patients died of SD, 4 patients experienced an appropriate ICD discharge, 7 patients died of stroke, 3 patients died of heart failure, and 2 patients died of post-procedural complications. The distribution of the causes of death is summarized in Figure 2. Independent predictors of the cardiovascular mortality events were a lower LVEDD at the last check-up ( $H R=0.90$, 95\% Cl: 0.82-0.99; $p=0.027)$ and a higher baseline maximum LVWT (HR = 1.3, 95\% Cl: 1.14-1.49; $p<0.001)$.

Sudden death events (SD, appropriate ICD discharge) occurred in eight patients (5.1\%, mean age

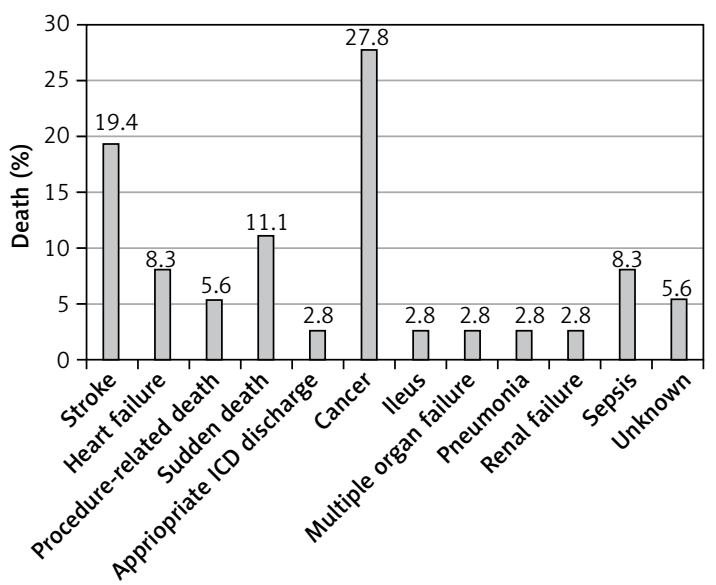

Figure 1. Causes of death at death: $74.9 \pm 3.4$ years). The annual sudden mortality rate and the annual sudden mortality rate including the first appropriate ICD discharge were $0.5 \%$ and $1.1 \%$, respectively. The only independent predictor of sudden mortality events was a higher baseline maximum LVWT $(\mathrm{HR}=1.37,95 \% \mathrm{Cl}$ : $1.12-1.69 ; p<0.001)$.

Non-cardiovascular mortality events occurred in 17 patients (44\% of all mortality events, $11 \%$ of all patients, mean age at death: $76 \pm 7$ years) with an annual mortality rate of $2.3 \%$. Among these patients, 10 patients died of cancer, 3 patients died of sepsis, 1 patient died of ileus, 1 patient died of pneumonia, one patient died of multiple organ failure, and 1 patient died of renal failure. In 2 patients ( $5 \%$ of all mortality events), the cause of death was unknown.

Survival free of all-cause mortality at 1, 5, and 10 years was $95 \%$ (95\% Cl: $91-98 \%), 80 \%(95 \% \mathrm{Cl}$ : 72-86\%), and 59\% (95\% Cl: 42-73\%), respectively. Compared with the expected mortality in the sex- and age-matched general population, patients $\geq 60$ years of age after ASA showed an increased mortality ( $p=0.016$; Figure 3 ). According to the multivariable analysis, independent predictors of all-cause mortality events were a lower LVEDD at the last check-up $(\mathrm{HR}=0.93,95 \% \mathrm{Cl}$ : 0.87-0.99; $p=0.017$ ) and a higher baseline maximum LVWT $(\mathrm{HR}=1.16,95 \% \mathrm{Cl}: 1.05-1.29 ; p<$ $0.001)$. Survival free of all-cause mortality combined with the first appropriate ICD discharge at 1 , 5, and 10 years was $95 \%$ (95\% Cl: $91-98 \%), 77 \%$ (95\% Cl: 68-84\%), and 55\% (95\% Cl: 39-68\%), respectively. This observed mortality was higher in comparison with the expected survival of the ageand sex-matched general population $(p<0.002$; Figure 4).

\section{Discussion}

This is the first international study to report the long-term survival and the causes of death in

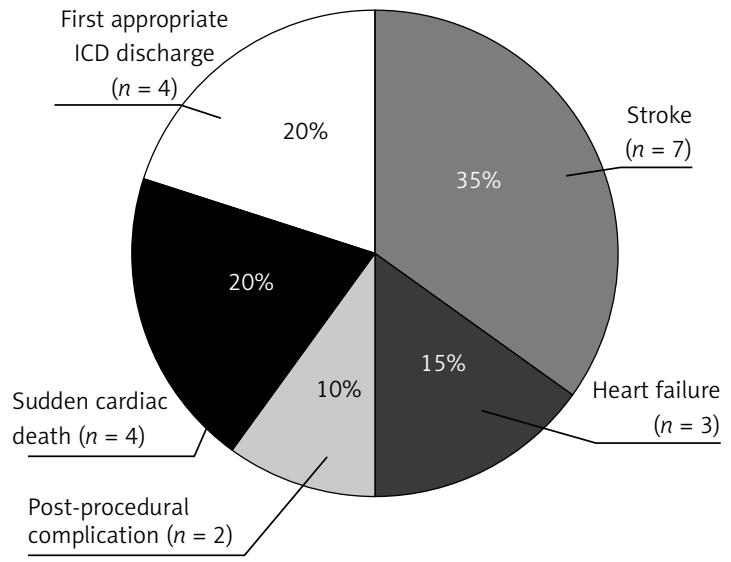

Figure 2. Cardiovascular causes of death 
the specific population of patients $\geq 60$ years of age with obstructive HCM who underwent ASA. The principal findings of the study are as follows: first, ASA was a safe procedure with low procedure-related mortality and led to an improvement in symptoms and a decrease in LVOG in patients $\geq 60$ years of age with obstructive HCM. Second, this study population showed a reduced life expectancy compared with the expected mortality of the age- and sex-matched general population. Third, mortality was almost equally due to cardiovascular and non-cardiovascular causes of death. Fourth, independent predictors of all-cause mortality were a lower LVEDD at the last clinical checkup and a higher baseline maximum LVWT.

In previously published registry data, cardiovascular mortality in HCM patients was predominantly determined by SD, stroke, and heart failure $[6,7$, 22-24], and in older patients ( $\geq 75$ years), stroke was the most frequent cardiovascular cause of death [6]. The distribution of cardiovascular causes of death in this study population was similar. The observed annual sudden mortality rate was

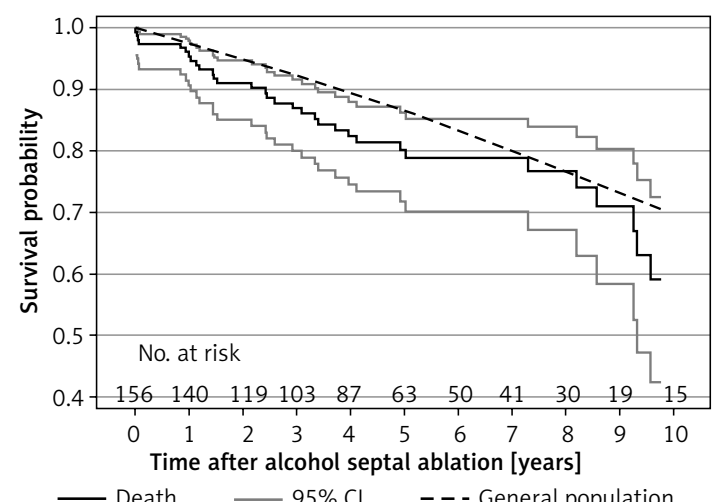

Figure 3. Kaplan-Meier survival curve describing all-cause mortality in patients with $\mathrm{HCM} \geq 60$ years of age after ASA compared with age- and sex-matched general population

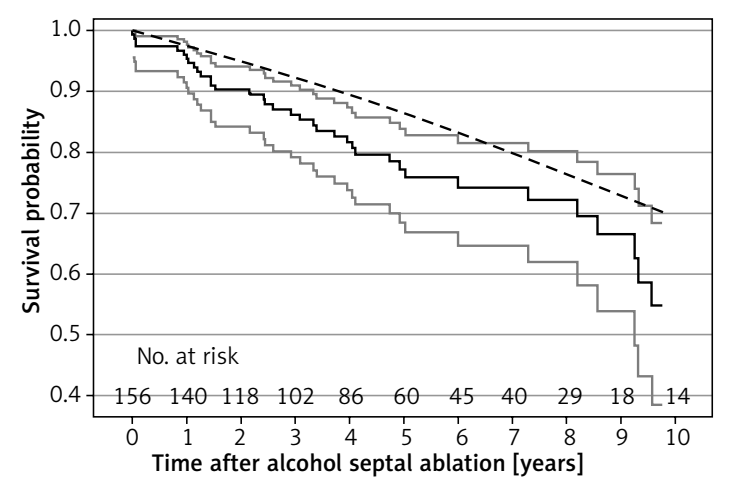

— Death/ICD discharge — $95 \% \mathrm{Cl}$ - - - General population

Figure 4. Kaplan-Meier survival curve describing the all-cause mortality and the first appropriate ICD discharge in patients with $\mathrm{HCM} \geq 60$ years of age after ASA compared with age- and sex-matched general population
$1.1 \%$, which is similarly low to the recently published data on ASA patients > 55 years of age described by Liebregts et al. [25] and to other previously published studies regarding HCM [15$18,25,26]$. In addition, the mean time between ASA and the first appropriate ICD discharge was $4 \pm 1.7$ years, which suggests a low arrhythmogenic potential of the resultant myocardial scar. The incidence of death due to heart failure was relatively low, which might suggest a protective effect of ASA. On the other hand, the incidence of stroke-related deaths was similarly high as in the previous reports $[6,7]$, which reminds us about the importance of the early initiation of anticoagulation therapy when appropriate [1, 2].

Baseline maximum LVWT is one of the established risk factors of SD in patients with HCM [1, $2,27]$. In the present study population, we confirmed the previously described observation that maximum LVWT is an independent predictor of SD mortality and all-cause mortality events [24, $28,29]$. This finding supports the importance of this risk factor in the assessment of the risk of SD. Lower LVEDD at the last check-up was found to be another independent predictor of cardiovascular and all-cause mortality.

The main limitation of this study is its retrospective and observational design.

Also, although this multicenter study was conducted in two neighboring countries, most of the data were obtained in centers within one country, which is why the mortality rate was compared with the mortality data of the age- and sexmatched general population in this more represented country. Even though the mean follow-up in the study population was 4.8 years, the number of patients followed up for more than 5 years was rather small. This may have influenced the reliability of the results. In the present study, we used a common definition of SD as an unexpected natural death within $1 \mathrm{~h}$ after witnessed collapse in a previously stable patient. In elderly patients, this definition may potentially yield false-positive results, since the patients may also die suddenly of causes of death not directly related to HCM, such as acute coronary syndrome, stroke, or abdominal aortic aneurysm rupture. Another limitation of our study may be the fact that not all ICD discharges in this setting would have resulted in deaths, which may have led to potential overestimation of risk.

In conclusion, in patients $\geq 60$ years of age with obstructive HCM, ASA was a safe and effective procedure in the long-term follow-up. This study population showed a reduced life expectancy compared to the age- and sex-matched general population. Mortality was almost equally due to cardiovascular and non-cardiovascular causes of death. 


\section{Acknowledgments}

This research was funded by $\mathrm{MH} C Z$ - DRO, University Hospital Motol, Prague, Czech Republic 00064203 and SVV-2013-266509 from the Charles University in Prague.

\section{Conflict of interest}

The authors declare no conflict of interest.

\section{References}

1. Gersh BJ, Maron BJ, Bonow RO, et al. 2011 ACCF/AHA guidelines for the diagnosis and treatment of hypertrophic cardiomyopathy. Circulation 2011; 124: e783-831.

2. Elliott PM, Anastasakis A, Borger MA, et al. 2014 ESC guidelines on diagnosis and management of hypertrophic cardiomyopathy. Eur Heart J 2014; 35: 2733-79.

3. Perrot A, Tomasov P, Villard E, et al. Mutations in NEBL encoding the cardiac Z-disk protein nebulette are associated with various cardiomyopathies. Arch Med Sci 2016; 12: 263-78.

4. Ziółkowska L, Petryka J, Boruc A, Kawalec W. Comparison of echocardiography with tissue Doppler imaging and magnetic resonance imaging with delayed enhancement in the assessment of children with hypertrophic cardiomyopathy. Arch Med Sci 2017; 13: 328-36.

5. Veselka J, Anavekar NS, Charron P. Hypertrophic obstructive cardiomyopathy. Lancet 2017; 389: 1253-67.

6. Maron BJ, Casey SA, Hauser RG, Aeppli DM. Clinical course of hypertrophic cardiomyopathy with survival to advanced age. J Am Coll Cardiol 2003; 42: 882-8.

7. Maron BJ, Rowin EJ, Casey SA, et al. Risk stratification and outcome of patients with hypertrophic cardiomyopathy $\geq 60$ years of age. Circulation 2013; 127: 585-93.

8. Maron MS, Olivotto I, Betocchi S, et al. Effect of left ventricular outflow tract obstruction on clinical outcome in hypertrophic cardiomyopathy. N Engl I Med 2003; 348: 295-303.

9. Seggewiss H, Rigopoulos A, Welge D, Ziemssen P, Faber L. Long-term follow-up after percutaneous septal ablation in hypertrophic obstructive cardiomyopathy. Clin Res Cardiol 2007; 96: 856-863.

10. Veselka J, Krejčí J, Tomašov P, Zemánek D. Long-term survival after alcohol septal ablation for hypertrophic obstructive cardiomyopathy: a comparison with general population. Eur Heart J 2014; 35: 2040-5.

11. Kuhn H, Lawrenz T, Lieder F, et al. Survival after transcoronary ablation of septal hypertrophy in hypertrophic obstructive cardiomyopathy (TASH): a 10 year experience. Clin Res Cardiol 2008; 97: 234-43.

12. Jensen MK, Prinz C, Horstkotte D, et al. Alcohol septal ablation in patients with hypertrophic obstructive cardiomyopathy: low incidence of sudden cardiac death and reduced risk profile. Heart 2013; 99: 1012-7.

13. Leonardi RA, Townsend JC, Patel CA, et al. Alcohol septal ablation for obstructive hypertrophic cardiomyopathy: outcomes in young, middle-aged, and elderly patients. Catheter Cardiovasc Interv 2013; 82: 838-45.

14. Nagueh SF, Groves BM, Schwartz L, et al. Alcohol septal ablation for the treatment of hypertrophic obstructive cardiomyopathy. A multicenter North American registry. J Am Coll Cardiol 2011; 58: 2322-8.

15. Sorajja P, Ommen SR, Holmes DR Jr, et al. Survival after alcohol septal ablation for obstructive hypertrophic cardiomyopathy. Circulation 2012; 126: 2374-80.
16. Liebregts M, Vriesendorp PA, Mahmoodi BK, Schinkel AF, Michels $M$, ten Berg JM. A systematic review and metaanalysis of long-term outcomes after septal reduction therapy in patients with hypertrophic cardiomyopathy. JACC Heart Fail 2015; 3: 896-905.

17. Cooper RM, Shahzad A, Stables RH. Intervention in HCM: patient selection, procedural approach and emerging techniques in alcohol septal ablation. Echo Res Pract 2015; 2: R25-35.

18. Leonardi RA, Kransdorf EP, Simel DL, Wang A. Meta-analyses of septal reduction therapies for obstructive hypertrophic cardiomyopathy: comparative rates of overall mortality and sudden cardiac death after treatment. Circ Cardiovasc Interv 2010; 3: 97-104.

19. Kim LK, Swaminathan RV, Looser P, et al. Hospital volume outcomes after septal myectomy and alcohol septal ablation for treatment of obstructive hypertrophic cardiomyopathy: US Nationwide Inpatient Database, 2003-2011. JAMA Cardiol 2016; 1: 324-32.

20. Cuisset T, Lefèvre T. Contemporary techniques for catheter-based intervention for hypertrophic obstructive cardiomyopathy. Eurolntervention 12 Suppl 2016; X: X44-X47.

21. Veselka J, Zemánek D, Fiedler J, Šváb P. Real-time myocardial contrast echocardiography for echo-guided alcohol septal ablation. Arch Med Sci 2009; 5: 271-2.

22. Veselka J, Krejči J, Tomašov $P$, et al. Survival of patients $\leq 50$ years of age after alcohol septal ablation for hypertrophic obstructive cardiomyopathy. Can J Cardiol 2014; 30: 634-8.

23. Veselka J, Zemánek D, Jahnlová D, et al. Risk and causes of death in patients after alcohol septal ablation for hypertrophic obstructive cardiomyopathy. Can J Cardiol 2015; 31: 1245-51.

24. Maron BJ, Olivotto I, Spirito P, et al. Epidemiology of hypertrophic cardiomyopathy-related death: revisited in a large non-referral-based patient population. Circulation 2000; 102: 858-64.

25. Liebregts M, Steggerda RC, Vriesendorp PA, et al. Longterm outcome of alcohol septal ablation for obstructive hypertrophic cardiomyopathy in the young and the elderly. JACC Cardiovasc Interv 2016; 9: 463-9.

26. Steggerda RC, Damman K, Balt JC, Liebregts $M$, ten Berg JM, van den Berg MP. Periprocedural complications and long-term outcome after alcohol septal ablation versus surgical myectomy in hypertrophic obstructive cardiomyopathy: a single-center experience. JACC Cardiovasc Interv 2014; 7: 1227-34.

27. Elliott PM, Poloniecki J, Dickie S, et al. Sudden death in hypertrophic cardiomyopathy: identification of high risk patients. J Am Coll Cardiol 2000; 36: 2212-8.

28. Spirito P, Bellone P, Harris KM, Bernabo P, Bruzzi P, Maron BJ. Magnitude of left ventricular hypertrophy and risk of sudden death in hypertrophic cardiomyopathy. N Engl J Med 2000; 342: 1778-85.

29. Jensen MK, Jacobsson L, Almaas V, et al. Influence of septal thickness on the clinical outcome after alcohol septal ablation in hypertrophic cardiomyopathy. Circ Cardiovasc Interv 2016; 9: e003214. 